\title{
Is Laparoscopic Complete Mesocolic Excision and Central Vascular Ligation Really Necessary for All Patients With Right-Sided Colon Cancer?
}

\author{
Gyung Mo Son ${ }^{1}$, In Young Lee ${ }^{1}$, Yoon Suk Lee ${ }^{2}$, Bong-Hyeon Kye ${ }^{3}$, Hyeon-Min Cho ${ }^{3}$, Je-Ho Jang ${ }^{4}$, \\ Chang-Nam Kim ${ }^{4}$, Kil Yeon Lee ${ }^{5}$, Suk-Hwan Lee ${ }^{6}$, Jun-Gi Kim ${ }^{2,7}$; On behalf of The Korean Laparoscopic \\ Colorectal Surgery Study Group \\ ${ }^{1}$ Department of Surgery, Pusan National University Yangsan Hospital, Pusan National University School of Medicine, Yangsan; ${ }^{2}$ Department of \\ Surgery, Seoul St. Mary's Hospital, College of Medicine, The Catholic University of Korea, Seoul; ${ }^{3}$ Division of Colorectal Surgery, Department \\ of Surgery, St. Vincent's Hospital, College of Medicine, The Catholic University of Korea, Suwon; ${ }^{4}$ Department of Surgery, Daejeon Eulji \\ Medical Center, Eulji University School of Medicine, Daejeon; ${ }^{5}$ Department of Surgery, Kyung Hee University College of Medicine, Seoul; \\ ${ }^{6}$ Department of Surgery, Kyung Hee University Hospital at Gangdong, Kyung Hee University School of Medicine, Seoul; ${ }^{7}$ Department of \\ Surgery, Pyeongtaek St. Mary's Hospital, Pyeongtaek, Korea
}

Colon cancer treatment is on the way to evolution over several decades. The minimally invasive surgery has improved postoperative short-term outcomes. Adjuvant chemotherapy has prolonged the survival of advanced colon cancer patients. Hohenberger proposed the noble concept of complete mesocolic excision (CME) which consists of 3 components: plane surgery, sufficient longitudinal bowel resection, and central vascular ligation (CVL). Mesocolic plane surgery shares the same surgical principle of total mesorectal excision, which is maintaining the intact mesothelial envelope. However, there remain debates about the extent of bowel resection and the level of CVL for maximizing lymph node dissection. There is no solid clinical evidence for the oncological necessity and benefit of extended radical dissection in right hemicolectomy. CME with CVL based on open surgery has been adopted in laparoscopic surgery. So, it is also necessary to look at how the CME could be transformed and successfully implanted in the laparoscopic era. Recent rapid advances in surgical technology and cancer biology are preparing for fundamental changes in cancer surgery. In this study, we reviewed the history, oncological necessity, and compatibility of CME for the right hemicolectomy in the laparoscopic era and outline the new perspectives on the evolution of cancer surgery.

Keywords: Colonic neoplasms; Colectomy; Mesocolon; Laparoscopy; Lymph node excision

\section{INTRODUCTION}

Colon cancer is one of the most common gastrointestinal cancers.

Received: Nov 5, 2021 - Revised: Nov 15, 2021 • Accepted: Nov 15, 2021 Correspondence to: Jun-Gi Kim, M.D., Ph.D., Emeritus Professor Department of Surgery, Seoul St. Mary's Hospital, College of Medicine, The Catholic University of Korea, 222 Banpo-daero, Seocho-gu, Seoul 06591, Korea

Tel: +82-2-2258-2876, Fax: +82-2-595-2822, E-mail: jgkim@catholic.ac.kr ORCID: https://orcid.org/0000-0002-4538-4736

\section{(C) 2021 The Korean Society of Coloproctology}

This is an open-access article distributed under the terms of the Creative Commons Attribution NonCommercial License (https://creativecommons.org/licenses/by-nc/4.0) which permits unrestricted noncommercial use, distribution, and reproduction in any medium, provided the original work is properly cited.
As national cancer screening program is widely conducted, the early diagnosis rate of colon cancer patients is increasing. In the past, more than half of the patients with colon cancer had lymph node metastasis; however recently, the proportion of stage I and II colon cancer patients has increased with early detection [1].

Colon cancer treatment has undergone rapid development over the past several decades. The minimally invasive surgery has improved postoperative short-term outcomes, such as pain, recovery of bowel movement, and length of hospital stay [2]. Adjuvant chemotherapy has prolonged the survival of stage III and stage II patients with high-risk factors [3].

In 1982, total mesorectal excision (TME) was presented by Bill Heald of the United Kingdom as meticulous sharp dissection along 
the embryologic plane to remove micrometastatic foci that had spread in the mesorectum. As a result, the local recurrence rate of rectal cancer, which previously reached $40 \%$, was reduced to less than $10 \%$. This has been accepted as the most important surgical revolution of the modern century, showing that oncologic benefits can be obtained through improvement of surgical quality in the field of rectal cancer [4].

In 2009, Hohenberger et al. [5] of Erlangen University in Germany proposed the noble concept of complete mesocolic excision (CME) which consists of 3 components: plane surgery, sufficient longitudinal bowel resection, and central vascular ligation (CVL).

Mesocolic plane surgery of CME shares the same surgical principle of TME, which is maintaining the intact mesothelial envelope surrounding the lymphatics to avoid the spread of cancer cells [5]. However, there remain debates about the extent of bowel resection and the level of CVL for maximizing lymph node dissection. There is no solid clinical evidence for the oncological necessity and benefit of extended radical dissection, including central lymph node dissection, in right hemicolectomy.

Originally, CME was performed on the basis of open surgery, which completely exposed the superior mesenteric vascular structure and removed the central lymph nodes. So, it is also necessary to look at how the CME could be transformed and successfully implanted in the laparoscopic era. Recently, cancer biology is on way of evolution to help us deeper understand the mechanism of cancer metastasis, which is gradually approaching as great wave of fundamental change in cancer surgery.

In this article, we reviewed the history, oncological necessity, and compatibility of CME for the right hemicolectomy in the laparoscopic era and outline the new perspectives on evolution of cancer surgery that reflect the advances in surgical technology and cancer biology.

\section{MESOCOLIC PLANE SURGERY}

CME as mesocolic plane surgery has similar concept to TME as maintaining the intact mesocolic plane using the sharp dissection along Toldt's fascia. In the pathologic study comparing the quality of mesocolic plane surgery, West et al. [5] and Philip Quirke [6] showed a statistically significant survival difference in the intact mesocolon group compared to the incomplete mesocolon group with exposure of the muscularis propria. Although high-quality evidence is lacking due to the paucity of randomized controlled trials (RCT) for CME, surgeons have strong beliefs about the importance of mesocolic plane surgery and its potential oncological benefits. In the meta-analysis, CME has continued to derive excellent oncological results (Table 1) [7-10].

In traditional colon cancer surgery, Turnbull's no-touch technique had been widely accepted as a principle of surgical treatment to prevent cancer cell spillage, even if there is no solid evidence supporting this inspirational principle. In surgical training, professor's emphasis had been placed on the embryological surgi-

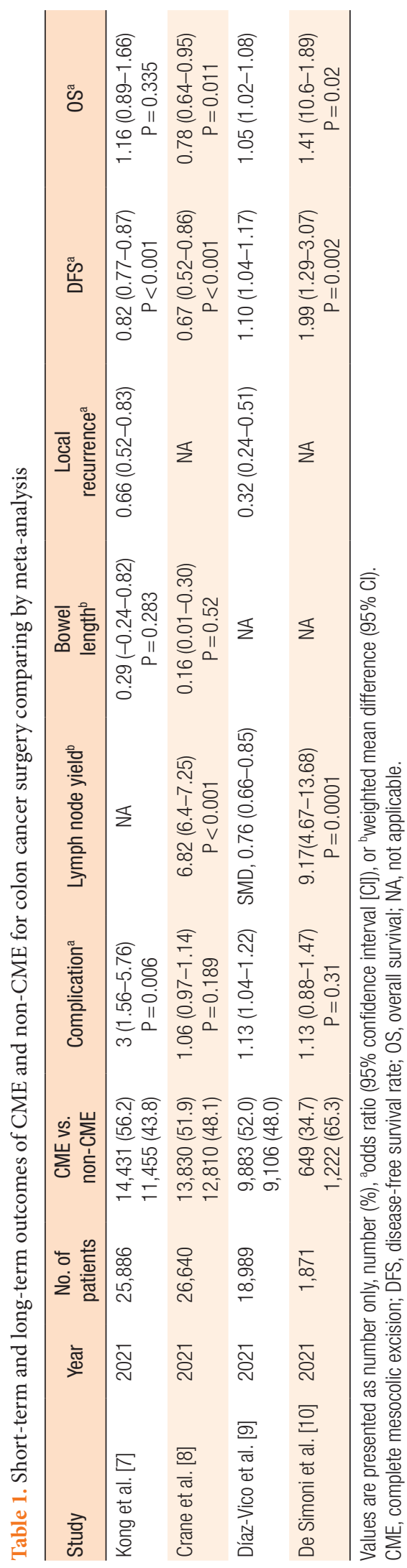


cal plan, Toldt's fascia, acting as a landmark for hemicolectomy. Born in Austria in 1840, Carl Florian Toldt was appointed professor of anatomy at the University of Vienna and later professor at the German University in Prague, Hungary. Over 100 years ago, Toldt asserted that the mesocolon could be separated from the retroperitoneum with a thin fascia [11]. However, Frederick Treves of the United Kingdom, a great leader in surgical anatomy at that time, argued that cadaveric research demonstrated that the mesocolon was merged into the retroperitoneum and could not be anatomically separated. The argument continued for a long time until the end of the 20th century. However, with the surgeon's recognition of the existence of the mesocolon, Toldt's fascia has maintained its reputation as a landmark of dissection for colectomy in the real surgical field. Recently, electron microscopy has proven that Toldt's fascia is composed of multiple mesothelial cell layers. Intensive studies by Cullingan et al. $[12,13]$ in Dublin, Ireland, on the structure of the mesocolon ended the 100-year debate between Toldt and Treves. Therefore, as Hogan and Winter [14] argued, CME had been built on the basis of a long history of colon surgery with careful mesocolic dissection along Toldt's fascia. However, it was only in the 21 st century that the surgical society coined the term CME as a novel and attractive nomenclature to describe the long tradition of mesocolic plane surgery.

The conceptualization of CME could provide standardized surgical quality and enable objective analysis through quantification of surgical treatment. Mesocolic plane surgery enables the quantitative evaluation of surgical quality through pathological evaluation. The mesorectal dissection quality of TME can be evaluated by pathologists as an analysis index for surgical quality and as an indicator of the oncological prognosis [15]. Therefore, CME could be accepted as a pathologic assessment tool for the evaluation of oncologic dissection quality, and it is already serving as an opportunity to standardize and improve surgical quality. This can be said to be an inevitable developmental stage necessary for the improvement and standardization of surgery in the era of evidencebased medicine.

\section{LONGITUDINAL EXTENT OF COLON RESECTION}

Hohenberger's CME could secure a proximal resection margin of approximately $10 \mathrm{~cm}$ from the ileocecal valve and a distal resection margin of $10 \mathrm{~cm}$ or more on the distal side of the tumor to excise sufficient longitudinal length of colon [14].

Radical resection was based on the long-standing surgeon's belief of the curative surgery with sufficient longitudinal margin. Grinnell [16], a surgeon and pathologist working at Columbia University in New York in the 1940s, proved the existence of tumor emboli in the lymphatic channels as a pathological tissue and confirmed the spread of cancer cells along the lymphatic chains. Furthermore, when the lymph nodes around the cancer were occupied by cancer cells and the lymphatic flow was blocked, retrograde lym- phatic backflow occurred through the paracolic lymph node, and metastasis could reach the distant paracolic lymph node from primary tumors more than $10 \mathrm{~cm}$. Therefore, vertical lymphatic flow toward the apical lymph node as well as retrograde horizontal lymphatic flow along the longitudinal axis of the colon was suggested as important pathways for lymph node metastasis [17].

Due to the observation of these lymph node metastasis patterns, it was argued that it was crucial to secure sufficient proximal and distal resection margins for radical resection and to perform high ligation along the feeding vessel as much as possible. This anatomical and pathological study of colorectal cancer metastasis pattern raised great expectations that curative surgery could be possible with extended radical resection for cancer patients suffering from a very low survival rate in the early 20th century. The expectation and hope for such a curative cancer operation led to enthusiasm for extended radical resection among brave and passionate surgeons. However, in the 1960s, the long-term survival results of extended radical resection were reported as comparable to those of segmental resection for colon cancer [18].

Disappointingly, there was no survival benefit of extended radical resection. In particular, most patients who had radical resection for the apical lymph node metastasis did not survive by critical cancer recurrence [19]. Based on these disappointing long-term survival results, central lymph node involvement was considered as the gate of systemic metastasis in which surgical salvage was impossible. As a result, when extended radical resection failed to salvage colon cancer patients, surgeons' enthusiasm for extended radical resection began to diminish in the Western surgical society.

CME with CVL and the Japanese D3 lymph node dissection are very similar concepts, but there is a difference of longitudinal length in the proximal and distal resection margins between the 2 surgical methods. In Japan, most metastatic foci were distributed within $5 \mathrm{~cm}$ of the tumor in the pathologic study of metastatic lymph node location, so it has been considered sufficient to secure a resection margin of $5 \mathrm{~cm}$ on both sides. Therefore, in pathologic specimens from Erlangen and Tokyo resected bowel length, mesenteric area, and harvested lymph nodes were higher in the German CME group than Japanese D3 group. However, there was no difference in the long-term oncologic outcomes of colorectal cancer between the 2 study groups [20,21]. In addition, there are many studies that showed that there was no significant difference in the therapeutic effect of conventional radical resection and extended radical resection for colon cancer surgery [22-25].

Therefore, maintaining the intact mesocolic plane in CME is accepted as an important factor, but long resection margin greater than $10 \mathrm{~cm}$ from the primary tumor can be considered selectively depending on the location of the tumor, its relationship to the feeding vessel, and the spread of lymph node metastasis (Fig. 1).

\section{CENTRAL VASCULAR LIGATION}

CVL is performed for completion of mesocolon resection extend- 


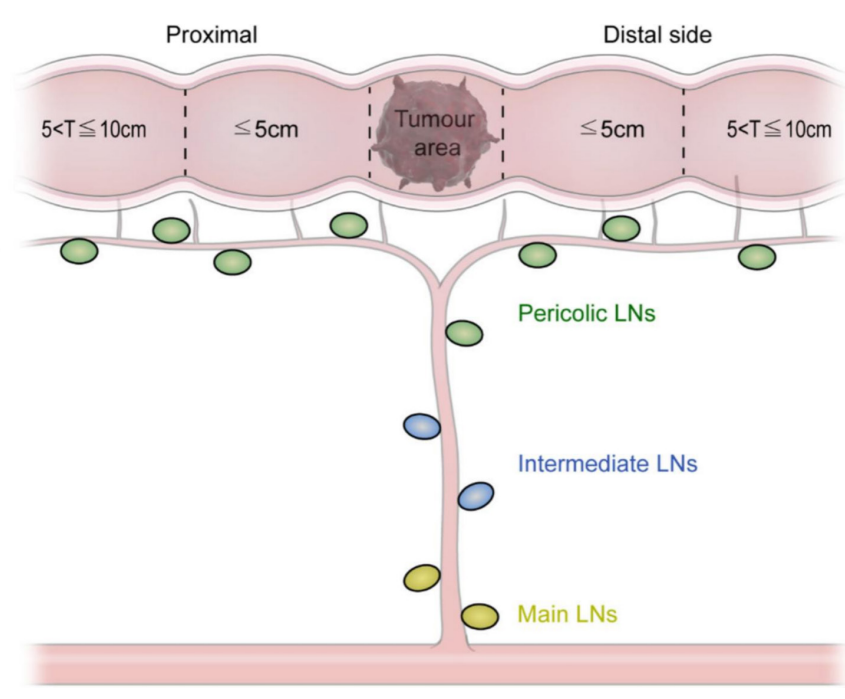

Fig. 1. Grouping of the retrieved lymph nodes (LN). Adapted from Yang et al. [31], according to the Creative Commons License of open access.

ing to mesenteric vascular root on the superior mesenteric artery (SMA) and superior mesenteric vein (SMV). For radical lymph node dissection, vascular ligation should be performed at the origin of the feeding arteries, the ileocolic, right colic, and middle colic arteries in right-sided colon cancer. This CVL is similar to the concept of D3 dissection in the Japanese Society of Cancer of the Colon and Rectum (JSCCR) guidelines [26].

The lymphatic pathway of the colon was published in detail by Jamieson and Dobson [27-29] at Leeds University in the United Kingdom in the early 20th century. They injected Prussian blue into the colon wall of cadavers and observed the dye flowing along the lymphatics and staining the lymph nodes. In these studies, they showed that colon cancer cells could spread out of the colon wall to regional lymph nodes, highlighting the need for radical lymph node dissection [27-29]. In a recent study using cadavers, Spasojevic et al. [30] divided the right, anterior, and posterior sections of D3 area around the SMV and SMA trunk to collect and count the associated lymph nodes. They found that 3 to 5 lymph nodes were present anterior and posterior to the SMV and SMA trunk, respectively. Therefore, as posterior dissection of the SMV and ileocolic artery retraction is required to remove all the main lymph nodes around the ileocolic artery root, the argument is that a more enlarged SMV dissection is necessary for true $\mathrm{D} 3$ dissection.

Unlike the manifest anatomical concept of CVL, the clinical definition of CVL is ambiguous and the surgical standard has not been established. The various surgical techniques used the different surgical landmarks to determine the boundary of main lymph node dissection depending on surgeon's expertise and preference. The level of CVL could be classified as the right side of the SMV, the interface between the SMV and SMA, or the left border of the SMA [31]. However, disagreements about the optimal CVL level to achieve patient safety and oncological radicality remain unresolved.

Interestingly, it has been reported that even if $\mathrm{CME}$ was described in the operation report, there were many cases in which true ligation was not achieved at the feeding artery root depending on the variation in the mesenteric vasculature. Kaye et al. [32] analyzed postoperative computed tomography (CT) scan to examine the remaining ileocolic artery stumps in patients who underwent right hemicolectomy. The arterial stump on CT angiography might be assumed that the apical lymph node dissection might be insufficient in some patients. Therefore, since true D3 dissection is sometimes really difficult to perform, most surgeons consider D3 dissection to be complete even if performing arterial ligation on the right side of the SMV, especially in laparoscopic CME.

In real surgical practice, the most common procedure implementing CVL is based on Gillot's concept of a surgical trunk of SMV. In 1964, Gillot [33] in France had named the area on the right side of the SMV between the gastrocolic trunk and the ileocolic vein as the surgical trunk as an anatomical landmark. Since lymphatics from the right colon collect in the surgical trunk and drain into the central lymphatic system, radical lymph node dissection for locoregional control that included the surgical trunk area was accepted. In 1995, Toyota et al. [34] explained that lymphatic flow in the right-sided colon forms a lymphatic chain on the right lateral side of the SMV that runs along the periportal lymphatics, with some lymphatic connections extending to the SMA lymph node at the root of the middle colic artery. Therefore, they said that D3 dissection can be sufficiently achieved by dissecting the central lymph node from the right lateral and anterior sides of the SMV.

Here, we can recognize that there is a practical gap between the anatomical concept of CVL and the clinically accepted scope of D3 dissection. It remains unclear whether it is sufficient to dissect the lymph nodes around the surgical trunk, or whether the extended dissection of both the anterior and posterior SMA is necessary for true D3 dissection. Until now, there is no solid clinical evidence for the oncological necessity and benefit of extended radical dissection, including central lymph node dissection, in right hemicolectomy (Fig. 2).

In the current ongoing RCTs, the definitions of D2 and D3 are almost the same as those in the JSCCR guidelines. In the COLD study, D3 node dissection is performed to expose the anterior surface of the SMV. In addition, vascular ligation should be performed at the arterial root by exposing the space between the SMA and SMV and performing SMA visualization. In the RELARC study, D3 dissection exposes the SMV anterior surface, but the boundary between the SMA and SMV is the delamination line. Therefore, anterior dissection of the SMA is not included in the D3 dissection. Even in this prospective study, the clear anatomical extent of D3 dissection is somewhat different. Nevertheless, surgeons expect that the long-term outcome of ongoing RCTs will provide a definite guideline for reasonable application of CVL in the near future (Table 2). 


\section{Annals of

Is Laparoscopic Complete Mesocolic Excision and Central Vascular Ligation Really Necessary for All

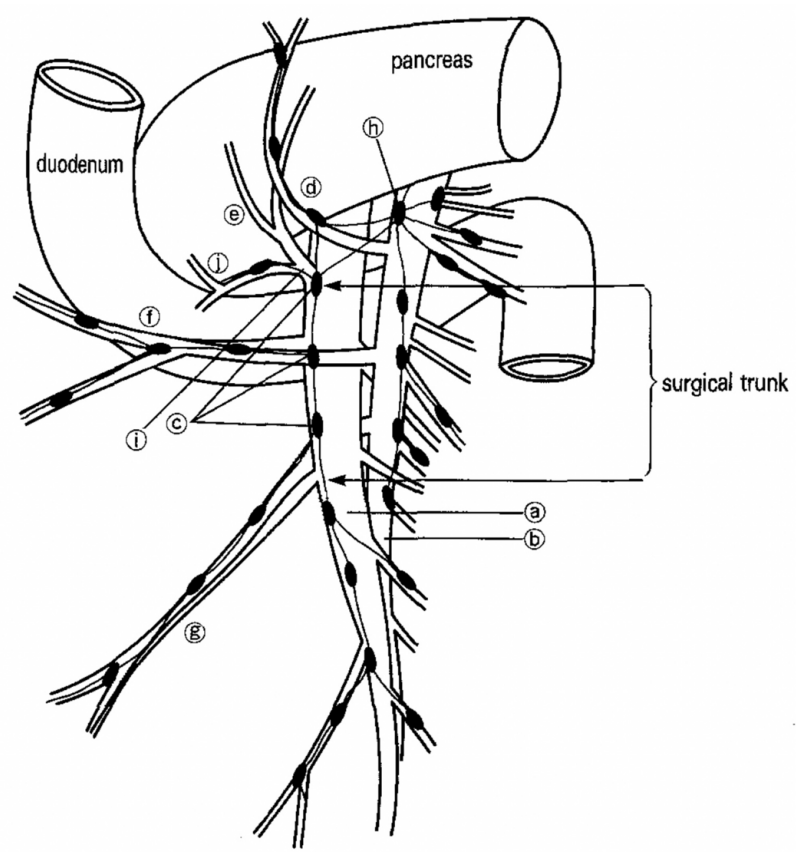

Fig. 2. Lymphatics of the right colon. a, superior mesenteric vein; b, superior mesenteric artery; c, main nodes; d, middle colic artery; e, middle colic vein; f, right colic artery and vein; g, ileocolic artery and vein; $h$, superior mesenteric node; i, Henle's gastrocolic trunk; $j$, right gastroepiploic vein. Adapted from Toyota et al. [34], with permission of Wolters Kluwer Health, Inc.

\section{Compatibility of laparoscopic CME with CVL}

Minimally invasive surgery has brought about a great change in the field of colon cancer surgery [35-37].

With laparoscopic or robotic surgery, faster postoperative recovery, reduced pain, and a quick return to daily life became possible. The large-scale RCTs, including the COST, COLOR, and MRCCLASICC trials, gave clear answers to questions about whether long-term oncological outcomes did not deteriorate after laparoscopic surgery. These studies proved that laparoscopic surgery is an oncologically safe procedure for colon cancer [38-40].

Actually, CME with CVL was designed as an open surgery because it requires extended radical vascular dissection. Hence, there are still doubts as to whether CME with CVL can be safely performed in laparoscopic surgery. Since the approach for mesenteric dissection and CVL in laparoscopic surgery is different from that of open surgery, various laparoscopic surgical techniques have been devised like a bottom-up, cranial-caudal, or arterial first approach [41-48]. In a recent meta-analysis, laparoscopic CME had comparable outcomes in harvested lymph nodes, longitudinal bowel length, mesenteric area, and vertical margin to open CME. In addition, long-term survival was not different between laparoscopic and open CME. Therefore, laparoscopic CME could also be performed safely with acceptable oncological outcomes [49-51]. As the surgeons' understanding of mesenteric vascular variation

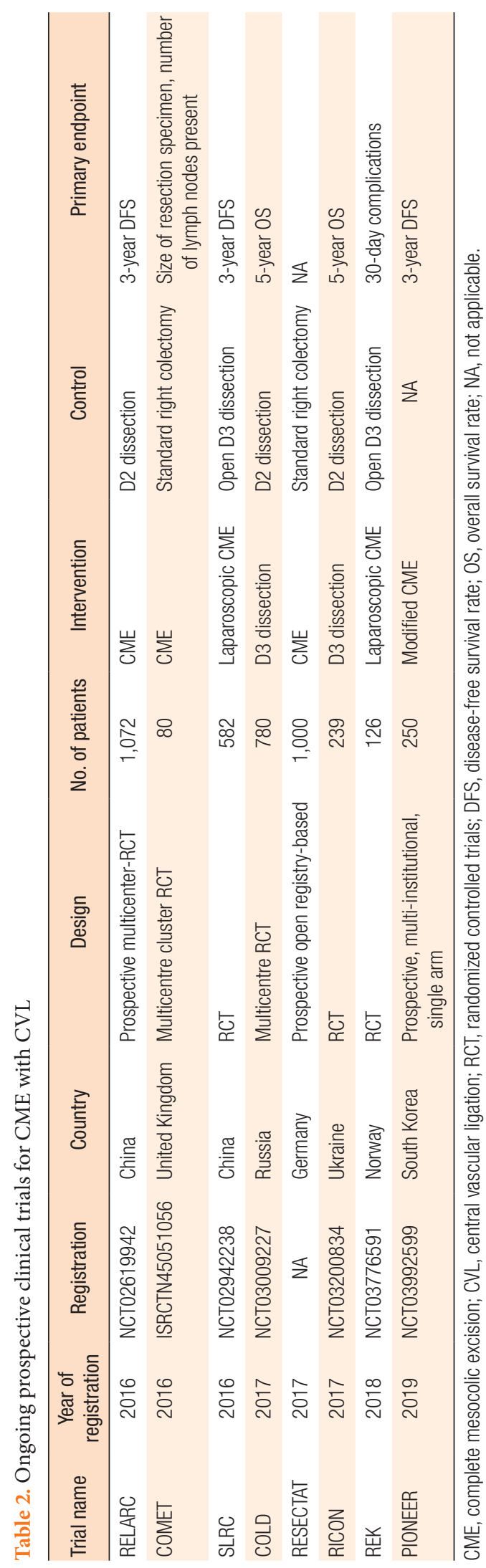


deepened and D3 dissection was actively attempted in the daily surgical practice field, it was found that various anomalies of the feeding artery and draining vein were quite common in the right colon region. The ileocolic artery is present in almost all patients; in $40 \%$, it passes anteriorly to the SMV and travels to the right and in $60 \%$, it branches from the SMA and proceeds posteriorly to the SMV [41, 52-54].

According to the definition of manifest $\mathrm{D} 3$ dissection in the JSCCR guidelines and the concept of CME with CVL, vascular ligation should be performed at the root of the feeding artery. If the ileocolic artery proceeds posteriorly to the SMV, the ileocolic root could be found after drastic retraction of fully mobilized SMV. Since laparoscopic surgery approaches the anterior chamber of the mesentery, dissection of the central lymph node anterior to the SMV and SMA can be performed relatively easily. Ligation of the ileocolic arterial root can be performed where the artery crosses the anterior chamber of SMV. However, dissecting the posterior SMV, retracting it to the left, and removing the central lymph node posterior to the SMV is a quite difficult operation. Dissection to the posterior surface of the SMV must be a dangerous operation that can cause crucial vascular injury $[55,56]$. In a recent study, the risk of vascular injury was 3-fold higher in the CME surgical group with full exposure to SMV [57]. Furthermore, the excessive SMA denuding can also cause damage to the autonomic nerve plexus supplying the small intestine, resulting in postoperative sequelae, such as diarrhea [34].

Because lymphatic flow in the right colon gathers from the surgical trunk on the right side of the SMV, and the lymph nodes anterior to the SMA are mainly connected with the lymphatics in the jejunum, SMA dissection beyond the anterior surface of the SMV is not required for lymph node dissection for right colon cancer [34]. Therefore, complete SMV dissection is usually beyond the scope of $\mathrm{D} 3$ lymph node dissection.

Literally, strict CVL can be detrimental overtreatment in patients without suspected metastatic lymph node on D3 area. That just increases the risk of vascular and neural damage to variant branch complex of the SMV. Furthermore, there is no clear oncologic benefit for strict CVL as an essential procedure. Therefore, complete CVL should be performed selectively by experienced surgeons in patients with suspected metastasis on the central lymph node [58, 59].

\section{ONCOLOGICAL NECESSITY OF COMPLETE MESOCOLIC EXCISION WITH CENTRAL VASCULAR LIGATION}

The number and location of involved lymph nodes and the metastatic lymph node ratio are known to be significant prognostic factors. In Japan, after colon resection, a surgeon extracts the lymph nodes from the specimen and marks the location of the resection. In a study on the metastatic lymph node distributions, D3 lymph node involvement showed a poorer prognosis than D1 lymph nodes [60]. In contrast, in some studies, the number of lymph nodes involved reflected more important clinical prognosis than the location of lymph node metastasis. Most patients with central lymph node involvement already have paracolic lymph node metastasis, and in these cases, apical lymph node metastasis tends to be considered clinically distant metastasis [61]. Therefore, in the presence of multiple lymph node metastases, lymph node involvement number and location are considered as definite poor prognostic factor. Interestingly, single lymph node metastasis has a very good prognosis. Even if a single lymph node metastasis is skip metastasis in the main lymph node area, a good prognosis can be expected regardless of location. This is homogenous to surgical resection, which has a curative therapeutic effect in single distant metastasis [62].

Surgeons need to determine whether to expand lymph node dissection with preoperative imaging. The diagnostic accuracy of preoperative CT is limited in predicting lymph node metastasis. Although the size, round shape, and heterogeneous texture of the lymph nodes increase the likelihood of metastatic lymph nodes, CT scans are not reliable for preoperative lymph node staging because metastatic nodes smaller than 3 to $5 \mathrm{~mm}$ are also commonly found. As the depth of the tumor invasion increases, the risk of lymph node metastasis increases [63]. Preoperative CT has negligible accuracy in diagnosing $\mathrm{T} 1-2$; however, the diagnosis rate of T3-4 is quite reliable. Therefore, the preoperative T stage plays an important role in determining the extent of lymph node dissection. Since the probability of D3 lymph node metastasis in T3-4 is high as $10 \%$, the JSCCR guidelines recommend D3 dissection in T3-4 patients [27].

Although surgeons are currently performing radical lymph node resections, scientific evidence for the therapeutic benefit of extended radical lymph node dissection is lacking. The radicality of lymph node dissection can be assessed by the number of harvested lymph nodes along with the location of lymph node dissection. In patients with nonmetastatic colon cancer, the higher the number of harvested lymph nodes, the better the oncological prognosis. The ratio of the involved lymph nodes to the harvested lymph nodes was identified as a strong prognostic indicator in stage III patients $[64,65]$. Although it is true that the removal of more lymph nodes by extended radical lymph node dissection is reflecting favorable prognosis, it requires considerable caution in interpreting this correlation as a cause-and-effect relationship. The number of harvested lymph nodes is not only related to resection radicality but also to tumor factors such as tumor size, T status, lymphocyte infiltration, and poorly differentiated microsatellite instability. The size of the hospital, surgeon's expertise, and pathological evaluation protocol are also related. The patient's age, body mass index, and the presence of a chronic bowel infection or inflammatory condition also affect the harvested lymph nodes [66, 67]. It is interesting to note that the number of harvested lymph nodes is related to these various factors, and since these factors are also affecting prognosis, it is difficult to conclude that a large number of har- 
vested lymph nodes is the cause of the positive prognosis.

The scientific mechanism remains unclear to prove that a better oncological prognosis with an increase in the number of harvested lymph nodes. Until now, several possible reasons are being investigated.

First, the most notable reason was the effect of adjuvant chemotherapy on staging migration. The prognosis was better in the patient group with 20 or more resected lymph nodes than in patients with fewer than 5 harvested lymph nodes $[68,69]$. The pathological diagnosis of metastatic lymph nodes may not have been possible due to insufficient lymph node dissection, and thus the treatment opportunity for adjuvant chemotherapy may have been missed. Appropriate chemotherapy with sufficient lymph node dissection may lead to improved survival rates resulting from more accurate nodal staging. The effect of staging migration, which has been considered the most important cause to date, has been minimized in several studies [70]. A population-based colon cancer registry study in Canada reported that the number of harvested lymph nodes has increased in recent years, but the proportion of metastatic lymph nodes has not changed [68]. Therefore, the therapeutic effect of harvested lymph node numbers may be achieved by different mechanisms.

Second, radical resection may have a therapeutic effect by removing occult metastatic lymph nodes. In pathological evaluations of lymph nodes, metastasis of cancer cells is usually evaluated by hematoxylin and eosin staining in 1 to 2 sections. In this conventional pathological lymph node evaluation protocol, occult metastasis could not be detected. Nodes that appear negative for metastases in hematoxylin and eosin staining revealed isolated tumor cells or micrometastases in 30\% of cases when immunohistochemical staining using pan-cytokeratin was performed. Realtime polymerase chain reaction (RT-PCR) can increase occult metastatic lymph node detection by up to $40 \%$. However, the pathological methodology for occult metastatic lymph nodes detection is still diverse and not standardized. Additional analyses, such as immunofluorescence staining and RT-PCR, require a large amount of medical resources. Some studies show contradictory results about patient's prognoses with isolated tumor cells [71]. This is likely to be the result of a change in the level of oncological threat posed by the isolated tumor cells according to the patient's immune response, rather than the therapeutic effect of micrometastasis resection itself [72].

Third, harvested lymph node number can be interpreted as an indicator of the patient's immune response to colon cancer. The greater the lymphocyte infiltration in the tumor and the more tumor antigen expression relating to microsatellite instability, the greater the immune response and the immunological defense against cancer. A strong immune response may be manifested by an increase in the number of harvested lymph nodes after surgery [73]. Since various questions about the therapeutic effect of harvested lymph nodes remain, contemporary surgeons are still performing radical D3 dissection, and the oncological necessity of
CME with CVL remains valid to date.

\section{NOVEL TECHNOLOGY OF LYMPH NODE MAPPING}

Recently, fluorescence-guided lymph node mapping (FLNM) has been developed as a promising surgical approach for image-guided surgery and has been approved the effect of increasing the number of harvested lymph nodes. When fluorescence dye, indocyanine green (ICG), is injected into the colonic wall, it enters the lymphatic drainage system from the distal perivascular space [74]. In colon cancer surgery, FLNM helps the surgeon visualize the lymphatic pathway to perform a more accurate dissection of the lymph nodes, especially around the main surgical trunk. Interestingly, the FLNM technique can increase the total number of harvested lymph nodes $[75,76]$. When visualizing the lymph nodes and lymphatic drainage pathway directly, the surgeon can dissect lymph nodes much more radically. When a fluorescent lymph node is found in the extraterritorial area outside the conventional radical dissection plane, a surgeon can extend the dissecting plane selectively for complete fluorescent lymph node removal. From an oncological point of view, at least 12 lymph nodes should be retrieved to evaluate the pathological stage of cancer so that patients can receive appropriate chemotherapy after surgery. The FLNM procedure is beneficial in harvesting more than 12 lymph nodes, and it can function as a surgical safety tool for the retrieval of an adequate number of lymph nodes for proper pathological staging [77].

Sentinel lymph node (SLN) mapping is a preliminary procedure for determining radical or selective lymph node dissection. It is an established technique in breast cancer and malignant melanoma to reduce postoperative complications due to radical resection. The concept of the SLN is the initial drainage of hypothetical lymph nodes from primary cancer. In many fields of cancer treatment, radioisotopes, blue dyes, and fluorescence dyes are used. The success rate of lymph node mapping using blue dye or radioisotopes is not satisfactory [78]. With the development of near-infrared camera systems, FLNM has been adopted for the detection of SLNs. The success rate of FLNM through colonoscopic submucosal ICG injection was over $90 \%$ [77].

In breast cancer and malignant melanoma, lymphatic drainage has a conduit or funnel-shaped pattern passing through 1 or 2 sentinel nodes as the first echelon lymph nodes, and metastatic cancer cells can spread into the second and third echelon lymph nodes. However, in colon cancer, lymphatic flow can be spread through various routes through the complex lymphatic network rather than through a linear connection, SLN mapping has many limitations in assessing the overall condition of lymph node metastasis [79].

There are still many questions to be answered, but further advances in fluorescence image-guided surgery could establish the basis for SLN mapping and enable the determination of expansion of radical lymphadenectomy, especially in early colon cancer. 


\section{EVOLUTION OF CANCER METASTASIS THEORY}

The concept of cancer metastasis has rapidly evolved in recent years. According to the linear progression theory proposed by William Stewart Halsted more than 100 years ago, cancer cells of primary tumors reach the apical lymph node along the adjacent lymph node pathway, which leads to distant metastasis [80, 81]. This linear metastasis pathway has been established as a fundamental concept in the current TNM staging. It is also a prognostic factor that accurately reflects the prognosis of most patients. However, skip metastasis, which occurs in $4 \%$ to $5 \%$ of patients, is an exception to the linear progression model. It is difficult to explain why distant metastasis still occurs in $20 \%$ to $30 \%$ of patients undergoing successful radical resection in the early stage [82]. Although the linear metastasis model is a concept that supports the therapeutic effect of curative radical resection, the oncologic results that radical resection itself does not achieve superior oncological outcomes compared to segmental resection raises doubts about the drawback of the radical resection.

In recent studies on metastasis, a parallel progression theory has been proposed whereby cancer cell metastasis already occurs at an early stage [83]. In colon cancer, micrometastasis of lymph nodes, circulating cancer cells in the peripheral blood, and micrometastasis of bone marrow are also found in the early stage, and such early micrometastasis causes poor prognosis. In addition, by analyzing cancer cell lines of metastatic foci as well as primary tumors, it was found that they consist of polyclonal cancer cells, not monoclonal cell line. This means that the polyclonal cancer cells of the primary tumor undergo seeding in multiple, continuous waves of metastasis [84]. The fact that cancer cells constituting primary tumors and metastatic lesions are heterogenous, polyclonal populations with genetically variable mutations explains why surviving cancer cells cause recurrence even after chemotherapy.

This polyclonal cancer cell population is a phenomenon that results from parallel progression. The recent understanding of this mechanism of cancer metastasis has heralded a change in the future of cancer treatment. The role of extended radical resection, which is currently being applied for locoregional cancer control, could be changed to improve the accuracy of the molecular pathologic diagnosis. In addition, the evaluation of circulating tumor cells in peripheral blood, circulating cell-free DNA, and micrometastasis of the bone marrow can be used to predict cancer metastasis and prognosis, as well as characterizing heterogeneous cancer cells through single-cell DNA sequencing [85]. Furthermore, by analyzing various gene mutations in the polyclonal cancer cell population, strategies for patient-tailored treatment can be established.

\section{CONCLUSION}

In conclusion, CME as mesocolic plane surgery is considered as one of oncologic surgical principles. The anatomical landmark for CVL is ambiguous and the oncological benefit is not yet clear. The ongoing RCTs will provide the answer for the necessity and radicality of CVL. The advances in surgical technology and cancer biology will come to the surgical society as a great wave demanding fundamental reformation of radical cancer surgery in the near future.

\section{CONFLICT OF INTEREST}

No potential conflict of interest relevant to this article was reported.

\section{ACKNOWLEDGMENTS}

The Authors thank to all members of The Korean Laparoscopic Colorectal Surgery Study Group in the Korean Society of Coloproctology.

\section{REFERENCES}

1. Hur H, Oh CM, Won YJ, Oh JH, Kim NK. Characteristics and survival of korean patients with colorectal cancer based on data from the Korea Central Cancer Registry data. Ann Coloproctol 2018;34:212-21.

2. Park SJ, Lee KY, Lee SH. Laparoscopic surgery for colorectal cancer in Korea: nationwide data from 2013 to 2018. Cancer Res Treat 2020;52:938-44.

3. André T, Boni C, Navarro M, Tabernero J, Hickish T, Topham C, et al. Improved overall survival with oxaliplatin, fluorouracil, and leucovorin as adjuvant treatment in stage II or III colon cancer in the MOSAIC trial. J Clin Oncol 2009;27:3109-16.

4. MacFarlane JK, Ryall RD, Heald RJ. Mesorectal excision for rectal cancer. Lancet 1993;341:457-60.

5. Hohenberger W, Weber K, Matzel K, Papadopoulos T, Merkel S. Standardized surgery for colonic cancer: complete mesocolic excision and central ligation: technical notes and outcome. Colorectal Dis 2009;11:354-65.

6. West NP, Morris EJ, Rotimi O, Cairns A, Finan PJ, Quirke P. Pathology grading of colon cancer surgical resection and its association with survival: a retrospective observational study. Lancet Oncol 2008;9:857-65.

7. Kong JC, Prabhakaran S, Choy KT, Larach JT, Heriot A, Warrier SK. Oncological reasons for performing a complete mesocolic excision: a systematic review and meta-analysis. ANZ J Surg 2021; 91:124-31.

8. Crane J, Hamed M, Borucki JP, El-Hadi A, Shaikh I, Stearns AT. Complete mesocolic excision versus conventional surgery for colon cancer: a systematic review and meta-analysis. Colorectal Dis 2021;23:1670-86.

9. Díaz-Vico T, Fernández-Hevia M, Suárez-Sánchez A, García-Gutiérrez C, Mihic-Góngora L, Fernández-Martínez D, et al. Com- 
plete mesocolic excision and D3 lymphadenectomy versus conventional colectomy for colon cancer: a systematic review and meta-analysis. Ann Surg Oncol 2021;28:8823-37.

10. De Simoni O, Barina A, Sommariva A, Tonello M, Gruppo M, Mattara G, et al. Complete mesocolic excision versus conventional hemicolectomy in patients with right colon cancer: a systematic review and meta-analysis. Int J Colorectal Dis 2021;36:881-92.

11. Culligan K, Remzi FH, Soop M, Coffey JC. Review of nomenclature in colonic surgery: proposal of a standardised nomenclature based on mesocolic anatomy. Surgeon 2013;11:1-5.

12. Culligan K, Coffey JC, Kiran RP, Kalady M, Lavery IC, Remzi FH. The mesocolon: a prospective observational study. Colorectal Dis 2012;14:421-30.

13. Culligan K, Walsh S, Dunne C, Walsh M, Ryan S, Quondamatteo F, et al. The mesocolon: a histological and electron microscopic characterization of the mesenteric attachment of the colon prior to and after surgical mobilization. Ann Surg 2014;260:1048-56.

14. Hogan AM, Winter DC. Complete mesocolic excision (CME): a "novel" concept? J Surg Oncol 2009;100:182-3.

15. Nagtegaal ID, van de Velde CJ, van der Worp E, Kapiteijn E, Quirke P, van Krieken JH, et al. Macroscopic evaluation of rectal cancer resection specimen: clinical significance of the pathologist in quality control. J Clin Oncol 2002;20:1729-34.

16. Grinnell RS. Lymphatic metastases of carcinoma of the colon and rectum. Ann Surg 1950;131:494-506.

17. Grinnell RS. The spread of carcinoma of the colon and rectum. Cancer 1950;3:641-52.

18. Grinnell RS. Lymphatic block with atypical and retrograde lymphatic metastasis and spread in carcinoma of the colon and rectum. Ann Surg 1966;163:272-80.

19. Grinnell RS. Results in the treatment of carcinoma of the colon and rectum; an analysis of 2,341 cases over a 35 year period with 5 year survival results in 1,667 patients. Surg Gynecol Obstet 1953; 96:31-42.

20. West NP, Kobayashi H, Takahashi K, Perrakis A, Weber K, Hohenberger $\mathrm{W}$, et al. Understanding optimal colonic cancer surgery: comparison of Japanese D3 resection and European complete mesocolic excision with central vascular ligation. J Clin Oncol 2012;30:1763-9.

21. Kobayashi H, West NP, Takahashi K, Perrakis A, Weber K, Hohenberger W, et al. Quality of surgery for stage III colon cancer: comparison between England, Germany, and Japan. Ann Surg Oncol 2014;21 Suppl 3:S398-404.

22. Morarasu S, Clancy C, Cronin CT, Matsuda T, Heneghan HM, Winter DC. Segmental versus extended colectomy for tumours of the transverse colon: a systematic review and meta-analysis. Colorectal Dis 2021;23:625-34.

23. Degiuli M, Reddavid R, Ricceri F, Di Candido F, Ortenzi M, Elmore U, et al. Segmental colonic resection is a safe and effective treatment option for colon cancer of the splenic flexure: a nationwide retrospective study of the Italian Society of Surgical Oncology-Colorectal Cancer Network Collaborative Group. Dis Colon
Rectum 2020;63:1372-82.

24. Hajibandeh S, Hajibandeh S, Hussain I, Zubairu A, Akbar F, Maw A. Comparison of extended right hemicolectomy, left hemicolectomy and segmental colectomy for splenic flexure colon cancer: a systematic review and meta-analysis. Colorectal Dis 2020;22:1885907.

25. Hacım NA, Akbaş A, Ulgen Y, Aktokmakyan TV, Meric S, Tokocin $\mathrm{M}$, et al. Influence of colonic mesenteric area on the number of lymph node retrieval for colon cancer: a prospective cohort study. Ann Coloproctol 2021 Sep 16 [Epub]. https://doi.org/10.3393/ ac.2021.00444.0063.

26. Tomita N, Ishida H, Tanakaya K, Yamaguchi T, Kumamoto K, Tanaka T, et al. Japanese Society for Cancer of the Colon and Rectum (JSCCR) guidelines 2020 for the clinical practice of hereditary colorectal cancer. Int J Clin Oncol 2021;26:1353-419.

27. Jamieson JK, Dobson JF. The lymphatics of the colon. Proc R Soc Med 1909;2(Surg Sect):149-74.

28. Jamieson JK, Dobson JF. VII. Lymphatics of the colon: with special reference to the operative treatment of cancer of the colon. Ann Surg 1909;50:1077-90.

29. Jamieson JK, Dobson JF. On the injection of lymphatics by Prussian blue. J Anat Physiol 1910;45(Pt 1):7-10.

30. Spasojevic M, Stimec BV, Dyrbekk AP, Tepavcevic Z, Edwin B, Bakka A, et al. Lymph node distribution in the $\mathrm{d} 3$ area of the right mesocolon: implications for an anatomically correct cancer resection: a postmortem study. Dis Colon Rectum 2013;56:1381-7.

31. Yang SY, Kim MJ, Kye BH, Han YD, Cho MS, Jeong SY, et al. Prospective study of oncologic outcomes after laparoscopic modified complete mesocolic excision for non-metastatic right colon cancer (PIONEER study): study protocol of a multicentre single-arm trial. BMC Cancer 2020;20:657.

32. Kaye TL, West NP, Jayne DG, Tolan DJ. CT assessment of right colonic arterial anatomy pre and post cancer resection: a potential marker for quality and extent of surgery? Acta Radiol 2016; 57:394-400.

33. Gillot C, Hureau J, Aaron C, Martini R, Thaler G, Michels NA. The superior mesenteric vein, an anatomic and surgical study of eighty-one subjects. J Int Coll Surg 1964;41:339-69.

34. Toyota S, Ohta H, Anazawa S. Rationale for extent of lymph node dissection for right colon cancer. Dis Colon Rectum 1995;38:70511.

35. Shah MF, Naeem A, Haq IU, Riaz S, Shakeel O, Panteleimonitis S, et al. Laparoscopy offers better clinical outcomes and long-term survival in patients with right colon cancer: experience from $\mathrm{Na}$ tional Cancer Center. Ann Coloproctol 2021 Jun 24 [Epub]. https: //doi.org/10.3393/ac.2021.00045.0006.

36. Sim JH, Jung EJ, Ryu CG, Paik JH, Kim G, Kim SR, et al. Shortterm outcomes of hand-assisted laparoscopic surgery vs. open surgery on right colon cancer: a case-controlled study. Ann Coloproctol 2013;29:72-6.

37. Cho JH, Lim DR, Hur H, Min BS, Baik SH, Lee KY, et al. Oncologic outcomes of a laparoscopic right hemicolectomy for colon 
cancer: results of a 3-year follow-up. J Korean Soc Coloproctol 2012;28:42-8.

38. Fleshman J, Sargent DJ, Green E, Anvari M, Stryker SJ, Beart RW $\mathrm{Jr}$, et al. Laparoscopic colectomy for cancer is not inferior to open surgery based on 5-year data from the COST Study Group trial. Ann Surg 2007;246:655-64.

39. Colon Cancer Laparoscopic or Open Resection Study Group, Buunen M, Veldkamp R, Hop WC, Kuhry E, Jeekel J, et al. Survival after laparoscopic surgery versus open surgery for colon cancer: long-term outcome of a randomised clinical trial. Lancet Oncol 2009;10:44-52.

40. Jayne DG, Thorpe HC, Copeland J, Quirke P, Brown JM, Guillou PJ. Five-year follow-up of the Medical Research Council CLASICC trial of laparoscopically assisted versus open surgery for colorectal cancer. Br J Surg 2010;97:1638-45.

41. Enomoto M, Katsumata K, Kasahara K, Tago T, Okazaki N, Wada $\mathrm{T}$, et al. Laparoscopic middle colic artery-preserved right hemicolectomy with true $\mathrm{D} 3$ lymph node dissection for right-sided colon cancer: modified complete mesocolic excision. Surg Endosc 2021; 35:2386-8.

42. Enomoto M, Katsumata K, Kasahara K, Tago T, Okazaki N, Wada $\mathrm{T}$, et al. Laparoscopic complete mesocolic excision with true central vascular ligation for right-sided colon cancer. Surg Endosc 2020;34:5640-1.

43. Sun Y, Yang HJ, Zhang ZC, Zhou YD, Li P, Zeng QS, et al. Fascial space priority approach for laparoscopic complete mesocolic excision (CME) plus central vascular ligation or extended lymphadenectomy (CVL/D3) in right-sided colon cancer (with video). Tech Coloproctol 2021 Oct 15 [Epub]. https://doi.org/10.1007/ s10151-021-02536-2.

44. Luo W, Li F, Xiao Y, Zhang X, Zhang L, Sun M, et al. Laparoscopic right hemicolectomy with complete mesocolic excision plus D3 lymphadenectomy $(\mathrm{CME}+\mathrm{D} 3)$ : a new medial approach for central vascular ligation. Updates Surg 2021 Aug 2 [Epub]. https:// doi.org/10.1007/s13304-021-01144-X.

45. Hernández P, de Nicolás L, Bodega I, Pastor C, Tejedor P. How to perform a laparoscopic right colectomy with complete mesocolic excision and central vascular ligation with intracorporeal anastomosis: a step-by-step superior mesenteric vein first approach-a video vignette. Colorectal Dis 2021;23:1606-7.

46. Suhardja TS, Das A, Rajkomar AK, Smart P, Heriot AG, Warrier SK. How to do a complete mesocolic excision and central vascular ligation. ANZ J Surg 2020;90:1469-71.

47. Luo W, Lu T, Xiao Y, Li F, Xu Z, Jia Y. A new medial-to-lateral approach for laparoscopic D3 lymphadenectomy plus complete mesocolic excision (D3 + CME) for right-sided colon cancer. Ann Surg Oncol 2021;28:3256-7.

48. Magouliotis DE, Baloyiannis I, Mamaloudis I, Bompou E, Papacharalampous C, Tzovaras GA. Laparoscopic versus open right colectomy for cancer in the era of complete mesocolic excision with central vascular ligation: pathology and short-term outcomes. J Laparoendosc Adv Surg Tech A 2021;31:1303-8.
49. Koc MA, Celik SU, Guner V, Akyol C. Laparoscopic vs open complete mesocolic excision with central vascular ligation for rightsided colon cancer. Medicine (Baltimore) 2021;100:e24613.

50. Franceschilli M, Di Carlo S, Vinci D, Sensi B, Siragusa L, Bellato V, et al. Complete mesocolic excision and central vascular ligation in colorectal cancer in the era of minimally invasive surgery. World J Clin Cases 2021;9:7297-305.

51. Wu C, Ye K, Wu Y, Chen Q, Xu J, Lin J, et al. Variations in right colic vascular anatomy observed during laparoscopic right colectomy. World J Surg Oncol 2019;17:16.

52. Ye K, Lin J, Sun Y, Wu Y, Xu J, He S. Variation and treatment of vessels in laparoscopic right hemicolectomy. Surg Endosc 2018; 32:1583-4.

53. Haywood M, Molyneux C, Mahadevan V, Lloyd J, Srinivasaiah N. The right colic artery: an anatomical demonstration and its relevance in the laparoscopic era. Ann R Coll Surg Engl 2016;98:560-3.

54. Lee SJ, Park SC, Kim MJ, Sohn DK, Oh JH. Vascular anatomy in laparoscopic colectomy for right colon cancer. Dis Colon Rectum 2016;59:718-24.

55. Pérez-Corbal L, Trujillo-Diaz JC, Alarcón I, Licardie E, Senent A, Morales-Conde $S$. Interactive 3D vascular reconstruction: a navigation tool to improve safety in laparoscopic D3 right colectomy. Colorectal Dis 2021;23:3030-2.

56. Xu L, Su X, He Z, Zhang C, Lu J, Zhang G, et al. Short-term outcomes of complete mesocolic excision versus $\mathrm{D} 2$ dissection in patients undergoing laparoscopic colectomy for right colon cancer (RELARC): a randomised, controlled, phase 3, superiority trial. Lancet Oncol 2021;22:391-401.

57. Thorsen Y, Stimec B, Andersen SN, Lindstrom JC, Pfeffer F, Oresland $\mathrm{T}$, et al. Bowel function and quality of life after superior mesenteric nerve plexus transection in right colectomy with D3 extended mesenterectomy. Tech Coloproctol 2016;20:445-53.

58. Paquette IM, Madoff RD, Sigurdson ER, Chang GJ. Impact of proximal vascular ligation on survival of patients with colon cancer. Ann Surg Oncol 2018;25:38-45.

59. Sammour T, Malakorn S, Thampy R, Kaur H, Bednarski BK, Messick CA, et al. Selective central vascular ligation (D3 lymphadenectomy) in patients undergoing minimally invasive complete mesocolic excision for colon cancer: optimizing the risk-benefit equation. Colorectal Dis 2020;22:53-61.

60. Balciscueta Z, Balciscueta I, Uribe N, Pellino G, Frasson M, García-Granero E, et al. D3-lymphadenectomy enhances oncological clearance in patients with right colon cancer: results of a metaanalysis. Eur J Surg Oncol 2021;47:1541-51.

61. Zenger S, Aytac E, Gurbuz B, Ozben V, Ozoran E, Baca B, et al. Metastasis to lymph nodes around the vascular tie worsens longterm oncological outcomes following complete mesocolic excision and conventional colectomy for right-sided colon cancer. Tech Coloproctol 2021;25:309-17.

62. Yeom SS, Lee SY, Kim CH, Kim HR, Kim YJ. The prognostic effect of adjuvant chemotherapy in the colon cancer patients with solitary lymph node metastasis. Int J Colorectal Dis 2019;34:1483- 
90.

63. Hong EK, Landolfi F, Castagnoli F, Park SJ, Boot J, Van den Berg J, et al. CT for lymph node staging of colon cancer: not only size but also location and number of lymph node count. Abdom Radiol (NY) 2021;46:4096-105.

64. Pyo JS, Shin YM, Kang DW. Prognostic implication of metastatic lymph node ratio in colorectal cancers: comparison depending on tumor location. J Clin Med 2019;8:1812.

65. Hao Y, Zhang J, Du R, Huang X, Li H, Hu P. Impact of negative lymph nodes on colon cancer survival and exploring relevant transcriptomics differences through real-world data analyses. Ann Transl Med 2019;7:525.

66. Willaert W, Mareel M, Van De Putte D, Van Nieuwenhove Y, Pattyn P, Ceelen W. Lymphatic spread, nodal count and the extent of lymphadenectomy in cancer of the colon. Cancer Treat Rev 2014; 40:405-13.

67. Altintas S, Bayrak M. Assessment of factors influencing lymph node count in colorectal cancer. J Coll Physicians Surg Pak 2019; 29:1173-8.

68. Baxter NN, Ricciardi R, Simunovic M, Urbach DR, Virnig BA. An evaluation of the relationship between lymph node number and staging in $\mathrm{pT} 3$ colon cancer using population-based data. Dis Colon Rectum 2010;53:65-70.

69. Tsai HL, Huang CW, Yeh YS, Ma CJ, Chen CW, Lu CY, et al. Factors affecting number of lymph nodes harvested and the impact of examining a minimum of 12 lymph nodes in stage I-III colorectal cancer patients: a retrospective single institution cohort study of 1167 consecutive patients. BMC Surg 2016;16:17.

70. Ning FL, Pei JP, Zhang NN, Wang J, Quan HG, Mei ZB, et al. Harvest of at least 18 lymph nodes is associated with improved survival in patients with $\mathrm{pN} 0$ colon cancer: a retrospective cohort study. J Cancer Res Clin Oncol 2020;146:2117-33.

71. Murray NP, Villalon R, Orrego S, Guzman E. The association of the neutrophil-lymphocyte ratio with the presence of minimal residual disease and outcome in patients with Stage II colon cancer treated with surgery alone. Colorectal Dis 2021;23:805-13.

72. Murray NP, Villalon R, Orrego S, Guzman E. Immune dysfunction as measured by the systemic immune-inflammation index is associated with the sub-type of minimal residual disease and outcome in stage II colon cancer treated with surgery alone. Asian Pac J Cancer Prev 2021;22:2391-7.

73. Murray NP, Villalon R, Hartmann D, Rodriguez MP, Aedo S. Improvement in immune dysfunction after FOLFOX chemotherapy for Stage III colon cancer is associated with improved minimal residual disease prognostic subtype and outcome. Colorectal Dis 2021;23:2879-93.

74. Soares AS, Lovat LB, Chand M. Intracorporeal lymph node mapping in colon cancer surgery. Eur J Surg Oncol 2019;45:2316-8.

75. Park SY, Park JS, Kim HJ, Woo IT, Park IK, Choi GS. Indocyanine green fluorescence imaging-guided laparoscopic surgery could achieve radical D3 dissection in patients with advanced right-sided colon cancer. Dis Colon Rectum 2020;63:441-9.

76. Son GM, Ahn HM, Lee IY, Ha GW. Multifunctional indocyanine green applications for fluorescence-guided laparoscopic colorectal surgery. Ann Coloproctol 2021;37:133-40.

77. Ahn HM, Son GM, Lee IY, Shin DH, Kim TK, Park SB, et al. Optimal ICG dosage of preoperative colonoscopic tattooing for fluorescence-guided laparoscopic colorectal surgery. Surg Endosc 2021 Feb 26 [Epub]. https://doi.org/10.1007/s00464-021-08382-5.

78. Saha S, Philimon B, Efeson M, Helina A, Elgamal M, Kiya G, et al. The role of sentinel lymph node mapping in colon cancer: detection of micro-metastasis, effect on survival, and driver of a paradigm shift in extent of colon resection. Clin Exp Metastasis 2021 Oct 26 [Epub]. https://doi.org/10.1007/s10585-021-10121-y.

79. Nissan A, Protic M, Bilchik A, Eberhardt J, Peoples GE, Stojadinovic A. Predictive model of outcome of targeted nodal assessment in colorectal cancer. Ann Surg 2010;251:265-74.

80. Patriarca C, Pini GM, Conti G. Invasion and metastasis: a historical perspective. Pathologica 2020;112:229-33.

81. Baum M. Modern concepts of the natural history of breast cancer: a guide to the design and publication of trials of the treatment of breast cancer. Eur J Cancer 2013;49:60-4.

82. Fortea-Sanchis C, Martínez-Ramos D, Escrig-Sos J. The lymph node status as a prognostic factor in colon cancer: comparative population study of classifications using the logarithm of the ratio between metastatic and nonmetastatic nodes (LODDS) versus the $\mathrm{pN}-\mathrm{TNM}$ classification and ganglion ratio systems. BMC Cancer 2018;18:1208.

83. Qiao L. Sentinel lymph node mapping for metastasis detection in colorectal cancer: a systematic review and meta-analysis. Rev Esp Enferm Dig 2020;112:722-30.

84. Naxerova K, Reiter JG, Brachtel E, Lennerz JK, van de Wetering M, Rowan A, et al. Origins of lymphatic and distant metastases in human colorectal cancer. Science 2017;357:55-60.

85. Ulintz PJ, Greenson JK, Wu R, Fearon ER, Hardiman KM. Lymph node metastases in colon cancer are polyclonal. Clin Cancer Res 2018;24:2214-24. 